Math. Model. Nat. Phenom.

Vol. 3, No. 1, 2008, pp. 27-54

\title{
Marangoni Convection in a Photo-Chemically Reacting Liquid
}

\author{
A.A. Golovin and V.A. Volpert ${ }^{1}$ \\ Department of Engineering Sciences and Applied Mathematics \\ Northwestern University, Evanston, IL 60208, USA
}

\begin{abstract}
Marangoni convection caused by a photochemical reaction of the type $A \stackrel{h \nu}{\rightleftharpoons} B$ in a deep liquid layer is studied. Linear stability analysis is performed and the conditions for Marangoni convection to occur are obtained. It is shown that increasing the rate of the direct reaction, for example, by increasing the light intensity, destabilizes the steady state and causes convective motion of the fluid, whereas increasing the rate of the inverse reaction stabilizes the steady state. A weakly nonlinear analysis of the problem is performed that gives conditions for hexagonal convective patters to occur. It is shown that, in the case of small light absorption length, the hexagonal cells correspond to "down"-hexagons.
\end{abstract}

Key words: Marangoni convection, photochemical reaction, stability analysis, nonlinear analysis

AMS subject classification: 76E06, 76E17, 76E30

\section{Introduction}

Marangoni convection in liquid layers exhibits complex patterns and spatio-temporal dynamics and has important applications in chemical engineering and materials processing, biology and medicine, microfluidics, etc. [1,2]. It occurs due to gradients of interfacial tension caused by gradients of temperature or concentration in the course of heat- or mass transfer. Marangoni convection is known to exhibit formation of spatio-temporal patterns with different symmetries: stationary patterns such as hexagons, rolls and squares $[3,4,5,6,7,8,9,10]$ as well as oscillatory patterns such as nonlinear waves and solitons $[11,12,13,14,15,16]$

\footnotetext{
${ }^{1}$ Corresponding author. Email: v-volpert@northwestern.edu
} 
(see also $[1,2]$ for review). In $[17,18,19]$ Marangoni convection induced by light was studied experimentally. In these works the light heating effect was used: the intensity of the incident light was spatially modulated in order to cause surface-tension gradients due to temperature variations. In $[20,21]$ the same mechanism of the light-induced Marangoni flows was used to control the dynamics of pattern formation. Note, however, that Marangoni convection can be caused by light irradiation due to a different mechanism. In [22] it was claimed that Marangoni convection could cause the occurrence of hexagonal patterns in a solid polymer film formed from a polymer solution under irradiation by UV light that sustained photopolymerization reaction. Indeed, if a liquid layer contains reactants that participate in a photochemical reaction induced by an incident light, the light absorption in the liquid layer will cause concentration gradients across the layer. If either reactants or products or both have surface-active properties these gradients can cause Marangoni convection similar to a situation with thermocapillary convection in a liquid layer heated from the bottom or from the top. Note that in this case the photochemically-induced Marangoni convection is caused by a uniform illumination. Thus, in the present paper we investigate Marangoni convection in a deep liquid layer caused by an isothermal photochemical reaction. Although light absorption in the liquid can cause thermal gradients across the liquid layer which can affect the conditions for Marangoni convection to occur, we neglect here the heating effect of light absorption and concentrate solely on the concentration-capillary effects caused by a photochemical reaction. We perform both linear and nonlinear analyses of the problem and determine the conditions for such type of Marangoni convection to occur as well as the type of the emerging convective patterns near the instability threshold.

\section{Mathematical Model}

We consider a deep liquid layer on a planar substrate. The liquid contains a species $A$. The layer is uniformly illuminated by light that triggers a reversible chemical reaction $A \stackrel{h \nu}{\rightleftharpoons} B$ (see $[23,24]$ for examples). The light absorption in the liquid results in concentration gradients across the layer. The liquid surface tension depends on the concentrations of the reactant $A$ and the product $B$. This can lead to the Marangoni convection. The effects of gravity and buoyancy are neglected.

The system of equations that governs the behavior of this system consists of the NavierStokes and continuity equations

$$
\begin{aligned}
& \frac{\partial \vec{V}}{\partial t}+(\vec{V} \cdot \nabla) \vec{V}=-\frac{1}{\rho} \nabla p+\nu \nabla^{2} \vec{V}, \\
& \nabla \cdot \vec{V}=0,
\end{aligned}
$$

and mass balances for the species $A$ and $B$ that account for the chemical reaction, diffusion of the species and mass transport by the liquid flow

$$
\frac{\partial A}{\partial t}+(\vec{V} \cdot \nabla) A=D_{A} \nabla^{2} A-k_{1} I A+k_{2} B
$$




$$
\frac{\partial B}{\partial t}+(\vec{V} \cdot \nabla) B=D_{B} \nabla^{2} B+k_{1} I A-k_{2} B
$$

Since we assume that the liquid layer is deep we neglect the effect of the bottom and consider the equations in the half-space

$$
-\infty<x, y<\infty, \quad-\infty<z<0
$$

for time $t>0$. Here the velocity $\vec{V}$ is

$$
\vec{V}=(u, v, w),
$$

$p$ is the pressure, $\rho$ is the density, $\nu$ is the kinematic viscosity, $D_{A}$ and $D_{B}$ are the diffusion coefficients of $A$ and $B$, respectively. The light intensity, $I$, satisfies the equation

$$
-\frac{d I}{d z}+\kappa I=0
$$

where $\kappa=\kappa_{0}+\alpha A$ is the absorption coefficient, and $\kappa_{0}$ and $\alpha$ are constants. We assume for simplicity that most of the light is absorbed by the solvent so that the light absorption coefficient does not depend on the concentration of $A$, i.e., $\alpha=0$ and $\kappa=\kappa_{0}$. Thus, the light intensity satisfies the Beer-Lambert law

$$
I(z)=I_{0} e^{\kappa_{0} z}
$$

where $I_{0}$ is the incident light intensity.

The boundary conditions are as follows. At the free surface of the liquid layer (liquid-gas interface), which is assumed non-deformable, we have

$$
z=0: \quad w=0, \quad \eta \frac{\partial u}{\partial z}=\frac{\partial \sigma}{\partial x}, \quad \eta \frac{\partial v}{\partial z}=\frac{\partial \sigma}{\partial y}, \quad \frac{\partial A}{\partial z}=0, \quad \frac{\partial B}{\partial z}=0 .
$$

Here $\eta=\rho \nu$ is the dynamic viscosity and $\sigma$ is the surface tension. The far-field boundary conditions, corresponding to the bottom of the liquid layer, are

$$
z=-\infty: \quad u=0, \quad v=0, \quad w=0, \quad A=A_{0}, \quad B=0 .
$$

From the boundary conditions at $z=0$ we derive another condition that we will use further. Differentiating the second condition in (2.2) with respect to $x$, the third condition with respect to $y$, adding them up and using the continuity equation (2.1b) yields

$$
z=0: \quad-\eta \frac{\partial^{2} w}{\partial z^{2}}=\nabla_{x y}^{2} \sigma, \quad \nabla_{x y}^{2} \equiv \frac{\partial^{2}}{\partial x^{2}}+\frac{\partial^{2}}{\partial y^{2}}
$$

Assuming that the surface tension is a linear function of the concentrations $A$ and $B$, we obtain from $(2.4)$

$$
z=0: \quad-\eta \frac{\partial^{2} w}{\partial z^{2}}=\frac{\partial \sigma}{\partial A} \nabla_{x y}^{2} A+\frac{\partial \sigma}{\partial B} \nabla_{x y}^{2} B
$$


We nondimensionalize the problem

$x \rightarrow \ell_{*} x, \quad y \rightarrow \ell_{*} y, \quad z \rightarrow \ell_{*} z, \quad t \rightarrow t_{*} t, \quad \vec{V} \rightarrow \frac{\ell_{*}}{t_{*}} \vec{V}, \quad A \rightarrow A_{0} A, \quad B \rightarrow A_{0} B, \quad p \rightarrow p_{*} p$,

with

$$
\ell_{*}=\frac{1}{\kappa_{0}}, \quad t_{*}=\frac{1}{D_{A} \kappa_{0}^{2}}, \quad p_{*}=\rho \kappa_{0}^{2} D_{A}^{2},
$$

to obtain

$$
\begin{aligned}
& \frac{\partial A}{\partial t}+(\vec{V} \cdot \nabla) A=\nabla^{2} A-\hat{k}_{1} e^{z} A+\hat{k}_{2} B \\
& \frac{\partial B}{\partial t}+(\vec{V} \cdot \nabla) B=\frac{1}{d} \nabla^{2} B+\hat{k}_{1} e^{z} A-\hat{k}_{2} B \\
& \frac{\partial \vec{V}}{\partial t}+(\vec{V} \cdot \nabla) \vec{V}=-\nabla p+\mathrm{Sc}^{2} \vec{V} \\
& \nabla \cdot \vec{V}=0, \\
& z=0: \quad \frac{\partial A}{\partial z}=\frac{\partial B}{\partial z}=0, \quad w=0, \\
& \frac{\partial u}{\partial z}=M_{A} \frac{\partial A}{\partial x}+M_{B} \frac{\partial B}{\partial x}, \quad \frac{\partial v}{\partial z}=M_{A} \frac{\partial A}{\partial y}+M_{B} \frac{\partial B}{\partial y}, \\
& \frac{\partial^{2} w}{\partial z^{2}}=-M_{A} \nabla_{x y}^{2} A-M_{B} \nabla_{x y}^{2} B, \\
& z=-\infty: \quad A=1, \quad B=0, \quad u=v=w=0 .
\end{aligned}
$$

Here

$$
\hat{k}_{1}=\frac{k_{1} I_{0}}{\kappa_{0}^{2} D_{A}}, \hat{k}_{2}=\frac{k_{2}}{\kappa_{0}^{2} D_{A}}, d=\frac{D_{A}}{D_{B}}, \mathrm{Sc}=\frac{\nu}{D_{A}}, M_{A}=\frac{A_{0}}{\kappa_{0} D_{A} \eta} \frac{\partial \sigma}{\partial A}, M_{B}=\frac{A_{0}}{\kappa_{0} D_{A} \eta} \frac{\partial \sigma}{\partial B},
$$

where Sc and $M_{A, B}$ are the Schmidt and Marangoni numbers, respectively. Since typically Marangoni convection is caused by surface-active properties of the dissolved components, one has $\partial \sigma / \partial A<0, \partial \sigma / \partial B<0$ and therefore, typically, $M_{A, B}<0$.

\section{Stationary Solution}

In this section we determine a stationary solution of the problem, which we denote by the subscript $s$. In this stationary state the liquid is quiescent, so that $\vec{V}_{s}=0, p_{s}=$ const, and the concentrations of the chemicals depend only on the depth $z$ of the layer. Thus, the concentrations of $A$ and $B$ satisfy the equations

$$
A_{s}^{\prime \prime}-\hat{k}_{1} e^{z} A_{s}+\hat{k}_{2} B_{s}=0,
$$




$$
\frac{1}{d} B_{s}^{\prime \prime}+\hat{k}_{1} e^{z} A_{s}-\hat{k}_{2} B_{s}=0
$$

which we solve on the semi-infinite interval $-\infty<z<0$ subject to the boundary conditions

$$
A_{s}^{\prime}(0)=0, \quad B_{s}^{\prime}(0)=0, \quad A_{s}(-\infty)=1, \quad B_{s}(-\infty)=0 .
$$

We remark that we can find a first integral of the system (3.7) by adding the two equations and integrating twice. Making use of the boundary conditions we obtain

$$
B_{s}=d\left(1-A_{s}\right) \text {. }
$$

Thus, eq. (3.7a) reduces to

$$
A_{s}^{\prime \prime}-\left(\hat{k}_{1} e^{z}+\hat{k}_{2} d\right) A_{s}=-\hat{k}_{2} d, \quad A_{s}^{\prime}(0)=0, \quad A_{s}(-\infty)=1 .
$$

The solution of this boundary value problem can be written in terms of Bessel functions. Indeed, the change of variables

$$
A_{s}(z)=a_{s}(\zeta), \quad \zeta=\exp \left(\frac{1}{2} z\right)
$$

in (3.8) yields

$$
\zeta \frac{d}{d \zeta}\left(\zeta \frac{d a_{s}}{d \zeta}\right)-\left(\zeta_{0}^{2} \zeta^{2}+\mu^{2}\right) a_{s}=-\mu^{2}, \quad a_{s}(0)=1, \quad a_{s}^{\prime}(1)=0
$$

where

$$
\zeta_{0}^{2}=4 \hat{k}_{1}, \quad \mu^{2}=4 \hat{k}_{2} d .
$$

We remark that for a fixed absorption coefficient $\kappa_{0}$ and fixed diffusive properties of the species, these two parameters can be treated as characterizing the rates of the direct and reverse reactions, respectively.

The homogeneous solution of the equation in (3.10) is a linear combination of the modified Bessel functions $I_{\mu}\left(\zeta_{0} \zeta\right)$ and $K_{\mu}\left(\zeta_{0} \zeta\right)$, and the solution of the boundary value problem (3.10) is

$$
a_{s}(\zeta)=\mu^{2}\left[I_{\mu}\left(\zeta_{0} \zeta\right) \int_{\zeta}^{1} \frac{1}{s} K_{\mu}\left(\zeta_{0} s\right) d s+K_{\mu}\left(\zeta_{0} \zeta\right) \int_{0}^{\zeta} \frac{1}{s} I_{\mu}\left(\zeta_{0} s\right) d s-C_{s} I_{\mu}\left(\zeta_{0} \zeta\right)\right]
$$

where $C_{s}$ is a constant given as

$$
C_{s}=\frac{K_{\mu}^{\prime}\left(\zeta_{0}\right)}{I_{\mu}^{\prime}\left(\zeta_{0}\right)} \int_{0}^{1} \frac{1}{s} I_{\mu}\left(\zeta_{0} s\right) d s
$$

The stationary solution $A_{s}(z)=a_{s}\left(e^{z / 2}\right)$ is shown in Fig.1. One can see the depletion layer near the free surface caused by the photochemical reaction. 


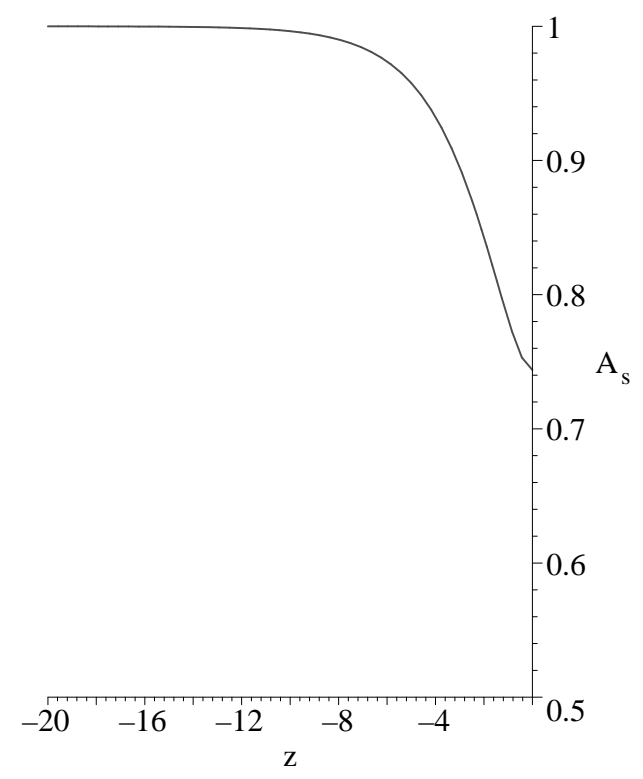

Figure 1: The exact solution of the stationary problem (3.8) as a function of $z$. Here $\mu=\zeta_{0}=1$, i.e. $\hat{k}_{1}=\hat{k}_{2} d=1 / 4$.

It is interesting to consider limiting cases in which a simple approximate solution can be derived. Since a photochemical reaction constant depends on the light intensity and can vary in a very wide range, as well as the light absorption length that strongly depends on the wavelength of the incident light, various limiting cases are physically relevant. For example, for $\zeta_{0} \ll 1$, which corresponds to the case when the light absorption length is much smaller than the reaction-diffusion length the solution can be written as

$$
a_{s}(\zeta)=1+a_{2 s}(\zeta ; \mu) \frac{\zeta_{0}^{2}}{\mu}+a_{4 s}(\zeta ; \mu) \frac{\zeta_{0}^{4}}{\mu^{2}}+\ldots
$$

where

$$
\begin{aligned}
& a_{2 s}(\zeta ; \mu)=\frac{\mu \zeta^{2}-2 \zeta^{\mu}}{4-\mu^{2}}, \\
& a_{4 s}(\zeta ; \mu)=\frac{\mu\left(\mu^{2}-16\right) \zeta^{\mu+2}+2 \mu^{2}(\mu+1) \zeta^{4}-\left(\mu^{3}+10 \mu^{2}-8 \mu-32\right) \zeta^{\mu}}{2(\mu+1)\left(\mu^{2}-4\right)\left(\mu^{2}-16\right)} .
\end{aligned}
$$

It turns out that even the first two terms in the above expansion provide an accurate approximation to the solution for $\zeta_{0}^{2} / \mu$ not being necessarily small. The accuracy of this approximation is illustrated in Fig. 2, where the two-term approximation, three-term approximation and the exact solution are plotted as a function of $\zeta$ for various parameter values. Both in Fig. 2(a) and Fig. 2(b) the value of the expansion parameter $\zeta_{0}^{2} / \mu$ is one. The solution in Fig. 2(b) is more accurate because the value of $\mu$ is larger - the error decreases with $\mu$. 


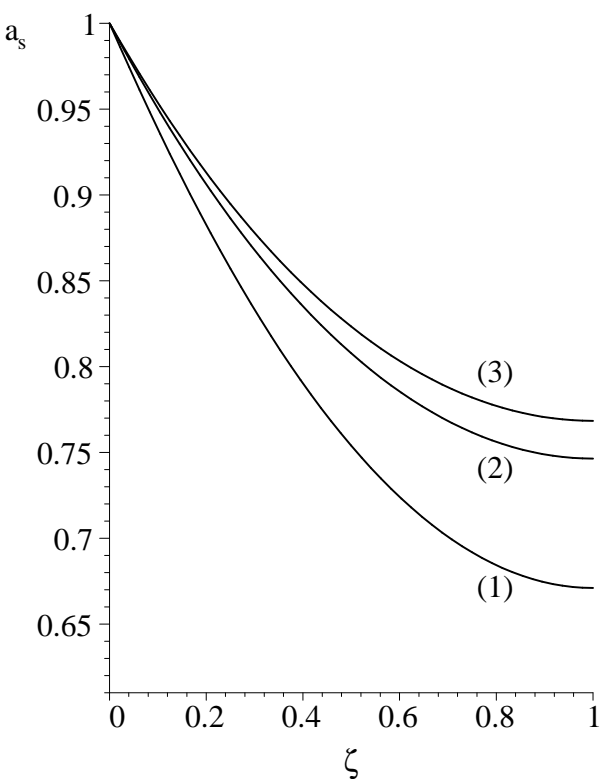

(a) $\zeta_{0}=1, \mu=1$

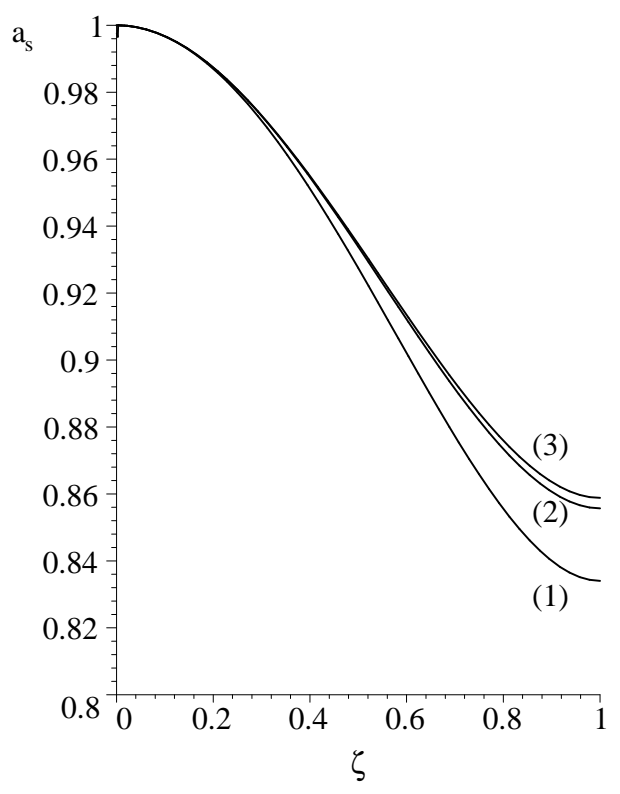

(b) $\zeta_{0}=2, \mu=4$

Figure 2: The solution of the problem (3.10). Here curves (1) and (2) are the graphs of the two- and three-term approximations in (3.13), respectively, while curve (3) is the exact solution (3.12).

For example, we can think of the magnitude of $a_{4 s}(\zeta ; \mu)$ as a measure of the accuracy of the two-term approximation. It turns out that the maximum of this function is attained at $\zeta=1$, and is quite small for all $\mu$. Indeed,

$$
a_{4 s}(1 ; \mu)=\frac{1}{(\mu+1)(\mu+4)} \leq \frac{1}{4},
$$

and the function decays sufficiently fast with $\mu$.

\section{Linear Stability Analysis}

We substitute

$$
A=A_{s}+\delta A_{p}, \quad B=B_{s}+\delta B_{p}, \quad \vec{v}=\delta \vec{v}_{p}, \quad p=p_{s}+\delta p_{p}
$$

into the system (2.6) and linearize it for small amplitude $\delta$. The subscript 'p' here stands for 'perturbation'. The linearized problem has the form

$$
\begin{aligned}
& \frac{\partial A_{p}}{\partial t}+w_{p} \frac{d A_{s}}{d z}=\nabla^{2} A_{p}-\hat{k}_{1} e^{z} A_{p}+\hat{k}_{2} B_{p}, \\
& \frac{\partial B_{p}}{\partial t}+w_{p} \frac{d B_{s}}{d z}=\frac{1}{d} \nabla^{2} B_{p}+\hat{k}_{1} e^{z} A_{p}-\hat{k}_{2} B_{p},
\end{aligned}
$$




$$
\begin{aligned}
& \frac{\partial \vec{V}_{p}}{\partial t}=-\nabla p_{p}+\operatorname{Sc} \nabla^{2} \vec{V}_{p}, \\
& \nabla \cdot \vec{V}_{p}=0 .
\end{aligned}
$$

Two comments are in place here. First, by applying the divergence operator to the equation (4.14c) and using the continuity equation (4.14d), we obtain the Laplace equation for the pressure perturbation,

$$
\nabla^{2} p_{p}=0
$$

Second, the equations for the horizontal components of the velocity perturbation, i.e., for $u_{p}$ and $v_{p}, \vec{V}_{p}=\left(u_{p}, v_{p}, w_{p}\right)$, decouple from the other equations. To study linear stability of the stationary solution, it is sufficient to consider the reduced system which includes equations (4.14a), (4.14b), (4.15) as well as the equation

$$
\frac{\partial w_{p}}{\partial t}=-\frac{\partial p_{p}}{\partial z}+\operatorname{Sc} \nabla^{2} w_{p}
$$

from the linearized Navier-Stokes equation (4.14c), but does not include the equations for $u_{p}$ and $v_{p}$. The boundary conditions are

$$
z=0: \quad \frac{\partial A_{p}}{\partial z}=0, \quad \frac{\partial B_{p}}{\partial z}=0, \quad w_{p}=0, \quad \frac{\partial^{2} w_{p}}{\partial z^{2}}=-M_{A} \nabla_{x y}^{2} A_{p}-M_{B} \nabla_{x y}^{2} B_{p}
$$

and

$$
z=-\infty: \quad A_{p}=0, \quad B_{p}=0, \quad w_{p}=0 .
$$

We consider the normal form perturbations

$$
\begin{aligned}
& A_{p}=\tilde{A}(z) \exp \left(\lambda t+i q_{x} x+i q_{y} y\right), \quad B_{p}=\tilde{B}(z) \exp \left(\lambda t+i q_{x} x+i q_{y} y\right), \\
& p_{p}=\tilde{p}(z) \exp \left(\lambda t+i q_{x} x+i q_{y} y\right), \quad w_{p}=\tilde{w}(z) \exp \left(\lambda t+i q_{x} x+i q_{y} y\right),
\end{aligned}
$$

which, upon substitution into the reduced system and the boundary conditions give the following linear problem

$$
\begin{aligned}
& \lambda \tilde{A}+\tilde{w} A_{s}^{\prime}=\tilde{A}^{\prime \prime}-q^{2} \tilde{A}-\hat{k}_{1} e^{z} \tilde{A}+\hat{k}_{2} \tilde{B}, \\
& \lambda \tilde{B}+\tilde{w} B_{s}^{\prime}=\frac{1}{d}\left(\tilde{B}^{\prime \prime}-q^{2} \tilde{B}\right)+\hat{k}_{1} e^{z} \tilde{A}-\hat{k}_{2} \tilde{B}, \\
& \tilde{p}^{\prime \prime}-q^{2} \tilde{p}=0, \\
& \lambda \tilde{w}=-\tilde{p}^{\prime}+\operatorname{Sc}\left(\tilde{w}^{\prime \prime}-q^{2} \tilde{w}\right), \\
& \tilde{A}^{\prime}(0)=0, \quad \tilde{B}^{\prime}(0)=0, \quad \tilde{w}(0)=0, \quad \tilde{w}^{\prime \prime}(0)=M_{A} q^{2} \tilde{A}(0)+M_{B} q^{2} \tilde{B}(0), \\
& \tilde{A}(-\infty)=0, \quad \tilde{B}(-\infty)=0, \quad \tilde{w}(-\infty)=0 .
\end{aligned}
$$


Here prime denotes the derivative with respect to $z, \lambda$ is the growth rate of the perturbation and $\left(q_{x}, q_{y}\right)$ is the wave vector, $q=\left(q_{x}^{2}+q_{y}^{2}\right)^{1 / 2}$.

In what follows we consider a simpler case, in which the diffusion coefficients of the two species are equal, i.e. $d=1$. Then adding up equations (4.17a) and (4.17b) and taking into account that for $d=1$

$$
A_{s}^{\prime}+B_{s}^{\prime}=0
$$

we obtain

$$
(\tilde{A}+\tilde{B})^{\prime \prime}-\left(q^{2}+\lambda\right)(\tilde{A}+\tilde{B})=0 .
$$

Solving the above equation for $\tilde{A}+\tilde{B}$ and applying the boundary conditions in (4.17e), (4.17f), we see that

$$
\tilde{A}+\tilde{B}=0 .
$$

Thus, eq. (4.17a) reduces to

$$
\tilde{A}^{\prime \prime}-\left(q^{2}+\hat{k}_{1} e^{z}+\hat{k}_{2}+\lambda\right) \tilde{A}=\tilde{w} A_{s}^{\prime}, \quad \tilde{A}^{\prime}(0)=0, \quad \tilde{A}(-\infty)=0 .
$$

Finally, we have obtained a system of equations (4.17c), (4.17d) and (4.18), with appropriate boundary conditions, for the unknown $\tilde{p}, \tilde{w}$ and $\tilde{A}$. The solutions of $(4.17 \mathrm{c})$ and $(4.17 \mathrm{~d})$ that satisfy the boundary conditions are

$$
\begin{aligned}
& \tilde{p}(z)=C e^{q z}, \\
& \tilde{w}(z)=\frac{q}{\lambda} C\left(e^{z \sqrt{q^{2}+\lambda / \mathrm{Sc}}}-e^{q z}\right),
\end{aligned}
$$

where $C$ is a constant. We remark that the singularity in $\tilde{w}$ at $\lambda=0$ is removable and in the limit $\lambda \rightarrow 0$

$$
\tilde{w}(z)=\frac{C}{2 \mathrm{Sc}} z e^{q z}
$$

a result that is needed to determine the stability boundary $\lambda=0$.

Next, we proceed to solving (4.18) for $\tilde{A}$. As in the case of the stationary problem, the solution of this boundary value problem can also be written in terms of Bessel functions. The change of variables (cf. (3.9))

$$
\tilde{A}(z)=C \tilde{a}(\zeta), \quad \zeta=\exp \left(\frac{1}{2} z\right)
$$

in (4.18) yields

$$
\zeta \frac{d}{d \zeta}\left(\zeta \frac{d \tilde{a}}{d \zeta}\right)-\left(\zeta_{0}^{2} \zeta^{2}+\beta^{2}\right) \tilde{a}=f(\zeta), \quad \tilde{a}(0)=0, \quad \tilde{a}^{\prime}(1)=0,
$$


where (cf. (3.11))

$$
\zeta_{0}^{2}=4 \hat{k}_{1}, \quad \beta^{2}=4 q^{2}+4 \hat{k}_{2}+4 \lambda,
$$

and the right-hand side $f(\zeta)$ is given by different expressions depending on whether $\lambda$ is or is not equal to zero (see (4.20) and (4.21)). If $\lambda \neq 0$, then

$$
f(\zeta)=\frac{2 q}{\lambda} \zeta \frac{d a_{s}(\zeta)}{d \zeta}\left[\zeta^{2 \sqrt{q^{2}+\frac{\lambda}{\mathrm{Sc}}}}-\zeta^{2 q}\right]
$$

and

$$
f(\zeta)=\frac{2}{\mathrm{Sc}} \zeta^{2 q+1} \frac{d a_{s}(\zeta)}{d \zeta} \ln \zeta
$$

for $\lambda=0$.

The solution of the boundary value problem (4.22) is

$$
\tilde{a}(\zeta)=-I_{\beta}\left(\zeta_{0} \zeta\right) \int_{\zeta}^{1} \frac{1}{s} K_{\beta}\left(\zeta_{0} s\right) f(s) d s-K_{\beta}\left(\zeta_{0} \zeta\right) \int_{0}^{\zeta} \frac{1}{s} I_{\beta}\left(\zeta_{0} s\right) f(s) d s+C_{p} I_{\beta}\left(\zeta_{0} \zeta\right),
$$

where $C_{p}$ is a constant given as

$$
C_{p}=\frac{K_{\beta}^{\prime}\left(\zeta_{0}\right)}{I_{\beta}^{\prime}\left(\zeta_{0}\right)} \int_{0}^{1} \frac{1}{s} I_{\beta}\left(\zeta_{0} s\right) f(s) d s
$$

Finally, we use the last boundary condition in (4.17e) to derive the dispersion relation. We observe that

$$
\tilde{B}(0)=-\tilde{A}(0)=-\tilde{a}(1)=\frac{1}{\zeta_{0} I_{\beta}^{\prime}\left(\zeta_{0}\right)} \int_{0}^{1} \frac{1}{s} I_{\beta}\left(\zeta_{0} s\right) f(s) d s
$$

and the dispersion relation takes the form

$$
\frac{M q S c}{\zeta_{0} I_{\beta}^{\prime}\left(\zeta_{0}\right)} \int_{0}^{1} \frac{1}{s} I_{\beta}\left(\zeta_{0} s\right) f(s) d s=1
$$

where

$$
M=M_{B}-M_{A} .
$$

At the stability boundary $\lambda=0$ we use (4.24) for $f(\zeta)$ so that (4.26) reduces to

$$
\frac{2 M q}{\zeta_{0} I_{\beta_{0}}^{\prime}\left(\zeta_{0}\right)} \int_{0}^{1} I_{\beta_{0}}\left(\zeta_{0} s\right) s^{2 q} \frac{d a_{s}}{d s} \ln s d s=1
$$




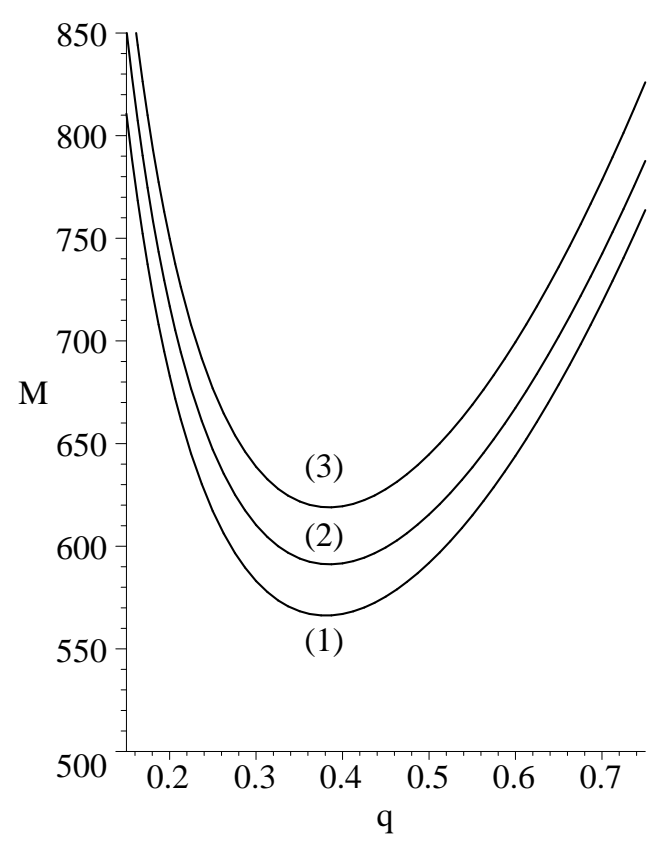

Figure 3: Neutral stability curves in the $(q, M)$ plane. Here $\zeta_{0}=0.5, \mu=1.5$. Curve (1) is given by the dispersion relation (4.28), curve (2) is given by (4.27) with $a_{s}$ being a two-term approximate solution (3.13), and curve (3) is given by (4.27) with $a_{s}$ being the exact solution $(3.12)$

where

$$
\beta_{0}^{2}=4 q^{2}+4 \hat{k}_{2}
$$

For small $\zeta_{0}$ the dispersion relation significantly simplifies. Using (3.13) we reduce the dispersion relation to

$$
M=\frac{\mu+2}{4 \zeta_{0}^{2}} \frac{\sqrt{\mu^{2}+4 q^{2}}}{q} \frac{\left(\sqrt{\mu^{2}+4 q^{2}}+2 q+2\right)^{2}\left(\sqrt{\mu^{2}+4 q^{2}}+2 q+\mu\right)^{2}}{2 \sqrt{\mu^{2}+4 q^{2}}+4 q+\mu+2} .
$$

Figure 3 shows an example of the neutral stability curve. The stationary solution is stable below the curve and unstable above it. The figure also demonstrates the accuracy of our approximate solutions. The neutral stability curve goes to infinity both as $q$ goes to zero and as $q$ goes to infinity. It can be shown that for all parameter values, not only for those used in the figure, the neutral stability curve has a single minimum. Indeed, let us denote the point of minimum by $\left(q_{c}, M_{c}\right)$. Figure 4 shows the location of the minimum of the neutral stability curve (4.28) as $\mu$ varies - the coordinates of the minimum increase with $\mu$. The coordinates of the minimum are found parametrically, with $\xi \equiv 2 q_{c} / \mu$ being the parameter. Indeed, (4.28) can be rewritten as

$$
M_{1}=8 \frac{1+\nu}{\nu^{4}} \frac{\sqrt{\xi^{2}+1}}{\xi} \frac{\left(\sqrt{\xi^{2}+1}+\xi+1\right)^{2}\left(\sqrt{\xi^{2}+1}+\xi+\nu\right)^{2}}{2 \sqrt{\xi^{2}+1}+2 \xi+\nu+1},
$$


where

$$
\xi=2 q_{c} / \mu, \quad \nu=2 / \mu, \quad M_{1}=M_{c} \zeta_{0}^{2} .
$$

The location of the minimum determined by

$$
\frac{d M_{1}}{d x}=0
$$

reduces to a quadratic equation for $\nu$. Solving this equation and finding $\nu$ and consequently $M_{1}$ and $q_{c}$ as a function of $\xi$, allows to plot the graph in Figure 4 . The calculation is straightforward but the resulting formulas are cumbersome and we omit them.

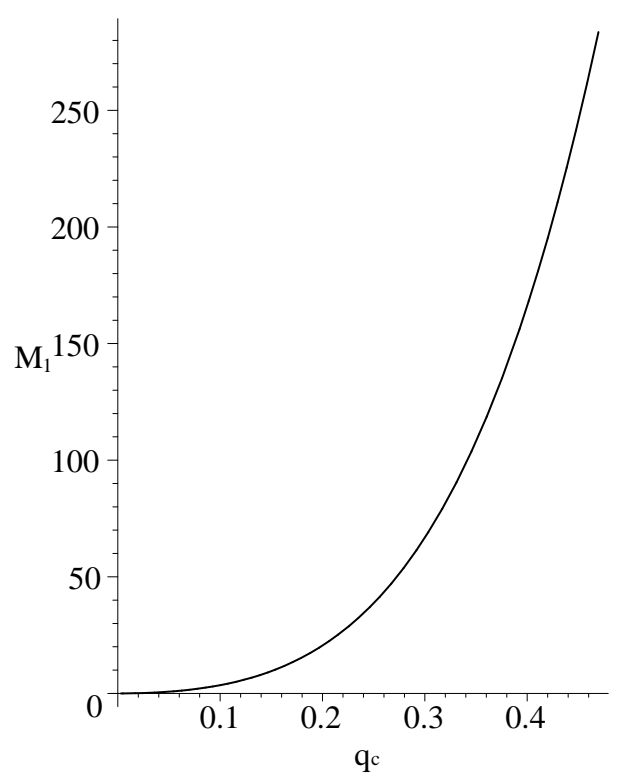

Figure 4: The location of the minimum of the neutral stability curve (4.28) as $\mu$ varies.

As one can see from the above analysis, the Marangoni instability occurs for $M=M_{B}-$ $M_{A}>M_{c}>0$. In the typical case of surface active reactants and products, $M_{A, B}<0$, this means that for Marangoni instability to occur one needs to have $\left|M_{A}\right|>\left|M_{B}\right|$ or $|\partial \sigma / \partial A|>|\partial \sigma / \partial B|$, i.e. the reactant must be more surface active than the product. In other words, if both the reactant and the product are surface active, surface-active property of the reactant destabilizes the system whereas that of the product stabilize it. This observation is the consequence of the concentration profiles of $A$ and $B$ in the steady state. Indeed, the concentration gradient of $A$ is negative ( $A$ is smaller closer to the interface) which corresponds to a typical unstable configuration in Marangoni convection: in this case convective flows near the interface, caused by interfacial fluctuations, bring more concentrated solution from below to the interface thus sustaining the fluctuations and the flow; this is similar to the typical Marangoni instability in a liquid layer heated from below. The concentration gradient of $B$ 
is, however, positive ( $B$ is larger closer to the interface). In this case convective flows bring less concentrated solution from below towards the interface, thus damping the interfacial fluctuations and the flow. This is similar to a typical stable configuration of a liquid layer heated from above. Note that if the only surface-active component is the reaction product, Marangoni convection cannot occur. (It can occur only in the case of surface-inactive product with $\partial \sigma / \partial B>0$ which is not typical).

As follows from (4.28), increasing $\mu$ stabilizes the steady state while increasing $\zeta_{0}$ destabilizes. In other words, increasing the rate of the direct reaction $A \rightarrow B$, for example, by increasing the light intensity, destabilizes the steady state, while increasing the rate of the inverse reaction stabilizes it. This is because the increase of the forward reaction rate leads to sharper concentration gradients near the interface while the increase of the rate of the inverse reaction reduces the concentration gradients.

\section{$5 \quad$ Weakly Nonlinear Analysis}

We now focus on the weakly nonlinear analysis of the system (2.6) in order to understand pattern formation near the instability threshold. Specifically, we study the appearance of hexagons and stripes.

Let the small parameter $\varepsilon$ characterize the magnitude of the bifurcating solution. We introduce the slow time $T=\varepsilon^{2} t$ and expand the unknowns $A, B, \vec{V}$ and $p$

$$
\begin{aligned}
A & =A_{s}+\varepsilon A_{1}+\varepsilon^{2} A_{2}+\varepsilon^{3} A_{3}+\ldots, \\
B & =B_{s}+\varepsilon B_{1}+\varepsilon^{2} B_{2}+\varepsilon^{3} B_{3}+\ldots, \\
\vec{V} & =\varepsilon \vec{V}_{1}+\varepsilon^{2} \vec{V}_{2}+\varepsilon^{3} \vec{V}_{3}+\ldots, \\
p & =p_{s}+\varepsilon p_{1}+\varepsilon^{2} p_{2}+\varepsilon^{3} p_{3}+\ldots
\end{aligned}
$$

As discussed below, the individual Marangoni numbers $M_{A}$ and $M_{B}$ enter the problem as a combination $M=M_{B}-M_{A}$, so that there is no need to consider them separately. Instead, we consider $M$, which is treated as the bifurcation parameter and expanded as

$$
M=M_{c}+\varepsilon^{2} m .
$$

Here $A, B, \vec{V}$ and $p$, as well as the coefficients in the above expansions of these quantities, are functions of $T, x, y$ and $z$.

Substituting the expansions into the system of equations (2.6) and collecting like powers of $\varepsilon$ we obtain at orders $\varepsilon^{j}(j=1,2,3)$ the sequence of problems

$$
\begin{aligned}
& \nabla^{2} A_{j}-\hat{k}_{1} e^{z} A_{j}+\hat{k}_{2} B_{j}-w_{j} \frac{d A_{s}}{d z}=R_{A j}, \\
& \nabla^{2} B_{j}+\hat{k}_{1} e^{z} A_{j}-\hat{k}_{2} B_{j}-w_{j} \frac{d B_{s}}{d z}=R_{B j},
\end{aligned}
$$




$$
\begin{aligned}
& \operatorname{Sc} \nabla^{2} \vec{V}_{j}-\nabla p_{j}=\vec{R}_{V j}, \\
& \nabla \cdot \vec{V}_{j}=0
\end{aligned}
$$

The right-hand sides $R_{A j}, R_{B j}, \vec{R}_{V j}$, for $j=1,2,3$ are given by

$$
\begin{aligned}
& R_{A 1}=0, \quad R_{B 1}=0, \quad \vec{R}_{V 1}=0, \\
& R_{A 2}=\left(\vec{V}_{1} \cdot \nabla\right) A_{1}, \quad R_{B 2}=\left(\vec{V}_{1} \cdot \nabla\right) B_{1}, \quad \vec{R}_{V 2}=\left(\vec{V}_{1} \cdot \nabla\right) \vec{V}_{1}, \\
& R_{A 3}=\frac{\partial A_{1}}{\partial T}+\left(\vec{V}_{1} \cdot \nabla\right) A_{2}+\left(\vec{V}_{2} \cdot \nabla\right) A_{1}, \quad R_{B 3}=\frac{\partial B_{1}}{\partial T}+\left(\vec{V}_{1} \cdot \nabla\right) B_{2}+\left(\vec{V}_{2} \cdot \nabla\right) B_{1}, \\
& \vec{R}_{V 3}=\frac{\partial \vec{V}_{1}}{\partial T}+\left(\vec{V}_{1} \cdot \nabla\right) \vec{V}_{2}+\left(\vec{V}_{2} \cdot \nabla\right) \vec{V}_{1} .
\end{aligned}
$$

The boundary conditions at $z=0$ for the concentration fields for $j=1,2,3$ are

$$
\frac{\partial A_{j}}{\partial z}=\frac{\partial B_{j}}{\partial z}=0
$$

and at $z=-\infty$ they are

$$
A_{j}=B_{j}=0
$$

Thus, one can see that $A_{j}+B_{j}=0, j=1,2,3$. This allows us to use the "composite" Marangoni number $M=M_{B}-M_{A}$ throughout the weakly nonlinear analysis and to eliminate $B_{j}$ from the problem. Applying the divergence operator to $(5.29 \mathrm{c})$ and using $(5.29 \mathrm{~d})$ we derive an equation for $p_{j}$.

Finally, the system of equations takes the form

$$
\begin{aligned}
& \nabla^{2} A_{j}-\left(\hat{k}_{1} e^{z}+\hat{k}_{2}\right) A_{j}-w_{j} \frac{d A_{s}}{d z}=R_{A j}, \\
& \operatorname{Sc} \nabla^{2} \vec{V}_{j}-\nabla p_{j}=\vec{R}_{V j}, \quad \vec{R}_{V j}=\left(R_{u j}, R_{v j}, R_{w j}\right), \\
& \nabla^{2} p_{j}=-\nabla \cdot \vec{R}_{V j} .
\end{aligned}
$$

The boundary conditions are

$$
\begin{aligned}
& z=0, \quad j=1,2: \quad \frac{\partial A_{j}}{\partial z}=0, \quad \frac{\partial u_{j}}{\partial z}=-M_{c} \frac{\partial A_{j}}{\partial x}, \quad \frac{\partial v_{j}}{\partial z}=-M_{c} \frac{\partial A_{j}}{\partial y} \\
& w_{j}=0, \quad \frac{\partial^{2} w_{j}}{\partial z^{2}}=M_{c} \nabla_{x y}^{2} A_{j}, \\
& z=0, \quad j=3: \quad \frac{\partial A_{3}}{\partial z}=0, \quad \frac{\partial u_{3}}{\partial z}=-\left(M_{c} \frac{\partial A_{3}}{\partial x}+m \frac{\partial A_{1}}{\partial x}\right)
\end{aligned}
$$




$$
\begin{aligned}
& \frac{\partial v_{3}}{\partial z}=-\left(M_{c} \frac{\partial A_{3}}{\partial y}+m \frac{\partial A_{1}}{\partial y}\right), \quad w_{3}=0, \quad \frac{\partial^{2} w_{3}}{\partial z^{2}}=M_{c} \nabla_{x y}^{2} A_{3}+m \nabla_{x y}^{2} A_{1}, \\
& z=-\infty, \quad j=1,2,3: \quad A_{j}=0, \quad u_{j}=v_{j}=w_{j}=0 .
\end{aligned}
$$

We now proceed to solving the above problems.

At $O(\varepsilon)$ we obtain the linearized system. The solution of the $O(\varepsilon)$ problem is

$$
\begin{aligned}
& p_{1}=e^{q_{c} z} E \\
& u_{1}=\frac{h^{\prime}(z)}{2 q_{c}^{2} \mathrm{Sc}} \frac{\partial E}{\partial x}, \quad v_{1}=\frac{h^{\prime}(z)}{2 q_{c}^{2} \mathrm{Sc}} \frac{\partial E}{\partial y}, \quad w_{1}=\frac{h(z)}{2 \mathrm{Sc}} E, \quad h(z)=z e^{q_{c} z}, \\
& A_{1}=A_{10}(z) E, \quad A_{10}(z)=\tilde{a}\left(e^{z / 2}\right) .
\end{aligned}
$$

Here $\tilde{a}(\zeta)$ is given by $(4.24),(4.25)$ with $\lambda=0$ and $q=q_{c}$. Next,

$$
E=L_{1} e_{1}+L_{2} e_{2}+L_{3} e_{3}+c . c ., \quad e_{1}=\exp \left(i q_{c} x\right), \quad e_{2,3}=\exp \left[i q_{c}\left(-\frac{x}{2} \pm \frac{\sqrt{3}}{2} y\right)\right]
$$

and c.c. denotes complex conjugate terms. The amplitudes $L_{1}, L_{2}, L_{3}$ are functions of the slow time $T$. We choose this form of the solution, with the three normal modes, because we want to describe the appearance of both hexagons and stripes as well as their interaction.

Next we turn to the $O\left(\varepsilon^{2}\right)$ problem. We introduce the quantities

$$
\begin{aligned}
& E_{1}=L_{1}^{2} e_{1}^{2}+L_{2}^{2} e_{2}^{2}+L_{3}^{2} e_{3}^{2}+c . c ., \quad E_{2}=2\left(\left|L_{1}\right|^{2}+\left|L_{2}\right|^{2}+\left|L_{3}\right|^{2}\right), \\
& E_{3}=L_{1} L_{2}^{*} e_{1} e_{2}^{*}+L_{1} L_{3}^{*} e_{1} e_{3}^{*}+L_{2} L_{3}^{*} e_{2} e_{3}^{*}+c . c ., \quad E_{4}=L_{1} L_{2} e_{3}^{*}+L_{1} L_{3} e_{2}^{*}+L_{2} L_{3} e_{1}^{*}+c . c .,
\end{aligned}
$$

where asterisk denotes the complex conjugate. We remark that

$$
E^{2}=E_{1}+E_{2}+2 E_{3}+2 E_{4}
$$

and

$$
\left(\frac{\partial E}{\partial x}\right)^{2}+\left(\frac{\partial E}{\partial y}\right)^{2}=q_{c}^{2}\left(-E_{1}+E_{2}-E_{3}+E_{4}\right)
$$

which allows us to write the right-hand sides of the equations in the form

$$
\begin{aligned}
& R_{u 2}=\frac{e^{2 q_{c} z}}{8 q_{c}^{2} \mathrm{Sc}^{2}} \frac{\partial S}{\partial x}, \quad R_{v 2}=\frac{e^{2 q_{c} z}}{8 q_{c}^{2} \mathrm{Sc}^{2}} \frac{\partial S}{\partial y}, \\
& S=-E_{1}+\left(1+4 q_{c} z+2 q_{c}^{2} z^{2}\right) E_{2}+\left(-1+2 q_{c} z+q_{c}^{2} z^{2}\right) E_{3}+\left(1+6 q_{c} z+3 q_{c}^{2} z^{2}\right) E_{4}, \\
& R_{w 2}=\frac{e^{2 q_{c} z}}{4 \mathrm{Sc}^{2}} z\left(1+q_{c} z\right)\left(2 E_{2}+E_{3}+3 E_{4}\right),
\end{aligned}
$$




$$
\begin{aligned}
& \nabla \cdot \vec{R}_{V 2}=\frac{e^{2 q_{c} z}}{8 \mathrm{Sc}^{2}}\left(4 E_{1}+4\left(1+4 q_{c} z+2 q_{c}^{2} z^{2}\right) E_{2}\right. \\
& \left.+\left(5+2 q_{c} z+q_{c}^{2} z^{2}\right) E_{3}+\left(5+18 q_{c} z+9 q_{c}^{2} z^{2}\right) E_{4}\right), \\
& R_{A 2}=\frac{e^{q_{c} z}}{4 \mathrm{Sc}}\left(\left[-2\left(1+q_{c} z\right) A_{10}(z)+2 z A_{10}^{\prime}(z)\right] E_{1}+\left[2\left(1+q_{c} z\right) A_{10}(z)+2 z A_{10}^{\prime}(z)\right] E_{2}\right. \\
& \left.+2\left[-\left(1+q_{c} z\right) A_{10}(z)+2 z A_{10}^{\prime}(z)\right] E_{3}+2\left[\left(1+q_{c} z\right) A_{10}(z)+2 z A_{10}^{\prime}(z)\right] E_{4}\right) .
\end{aligned}
$$

We remark that the terms proportional to $E_{4}$ are secular terms that appear in the $O\left(\varepsilon^{2}\right)$ problem due to resonance interactions of the modes (5.39). These secular terms are considered to be small and will contribute to the solvability condition at $O\left(\varepsilon^{3}\right)$ [25]. Specifically, secular terms in $R_{A 2}, R_{w 2}$ and $-\nabla \cdot \vec{R}_{V 2}$ are, respectively,

$$
\begin{aligned}
& S_{A 2}=\frac{1}{2 \mathrm{Sc}}\left[h^{\prime}(z) A_{10}(z)+2 h(z) A_{10}^{\prime}(z)\right], \\
& S_{w 2}=\frac{3}{4 \mathrm{Sc}^{2}} h^{\prime}(z) h(z), \\
& S_{p 2}=-\frac{1}{8 \mathrm{Sc}^{2}}\left[5\left(h^{\prime}\right)^{2}(z)+4 h^{\prime \prime}(z) h(z)\right] .
\end{aligned}
$$

Using the fact that $E_{1}, E_{2}$ and $E_{3}$ are eigenfunctions of the Laplacian $\nabla_{x y}^{2}$, specifically, that

$$
\nabla_{x y}^{2} E_{1}=-4 q_{c}^{2} E_{1}, \quad \nabla_{x y}^{2} E_{2}=0, \quad \nabla_{x y}^{2} E_{3}=-3 q_{c}^{2} E_{3}
$$

allows us to obtain the solution of the $O\left(\varepsilon^{2}\right)$ problem in the form

$$
\begin{aligned}
& p_{2}=p_{21} E_{1}+p_{22} E_{2}+p_{23} E_{3}, \\
& u_{2}=\frac{\partial Q}{\partial x}, \quad v_{2}=\frac{\partial Q}{\partial y} \\
& Q=Q_{1} E_{1}+Q_{2} E_{2}+Q_{3} E_{3}, \\
& w_{2}=w_{21} E_{1}+w_{22} E_{2}+w_{23} E_{3}, \\
& A_{2}=A_{21} E_{1}+A_{22} E_{2}+A_{23} E_{3},
\end{aligned}
$$

where the coefficients of $E_{1}, E_{2}$ and $E_{3}$ are functions of $z$ and are given in Appendix A.

We now turn to the $O\left(\epsilon^{3}\right)$ problem. Amplitude equations arise due to solvability conditions of the problem, which are needed because of the presence of secular terms in the right-hand sides. The right-hand side $R_{A 3}$ can be written in the form

$$
\begin{aligned}
& R_{A 3}=\left[S_{A 0} \frac{\partial L_{1}}{\partial T}+S_{A 3} L_{1}\left|L_{1}\right|^{2}+S_{A 4} L_{1}\left(\left|L_{2}\right|^{2}+\left|L_{3}\right|^{2}\right)\right] e_{1} \\
& +\left[S_{A 0} \frac{\partial L_{2}}{\partial T}+S_{A 3} L_{2}\left|L_{2}\right|^{2}+S_{A 4} L_{2}\left(\left|L_{1}\right|^{2}+\left|L_{3}\right|^{2}\right)\right] e_{2}
\end{aligned}
$$




$$
+\left[S_{A 0} \frac{\partial L_{3}}{\partial T}+S_{A 3} L_{3}\left|L_{3}\right|^{2}+S_{A 4} L_{3}\left(\left|L_{1}\right|^{2}+\left|L_{2}\right|^{2}\right)\right] e_{2}+\text { c.c. }+ \text { N.S.T. }
$$

where N.S.T. denotes non-secular terms that do not affect the solvability conditions, and the coefficients $S_{A 0}, S_{A 1}$ and $S_{A 2}$ are given by

$$
\begin{aligned}
& S_{A 0}=A_{10}, \\
& S_{A 3}=\frac{1}{2 \mathrm{Sc}}\left[2 h^{\prime} A_{21}+2 h A_{22}^{\prime}+h A_{21}^{\prime}+4 q_{c}^{2} A_{10} \mathrm{Sc} Q_{1}+2 A_{10}^{\prime} \mathrm{Sc} w_{21}\right], \\
& S_{A 4}=\frac{1}{4 \mathrm{Sc}}\left[3 h^{\prime} A_{23}+4 h A_{22}^{\prime}+2 h A_{23}^{\prime}+6 q_{c}^{2} A_{10} \mathrm{Sc} Q_{3}+4 A_{10}^{\prime} \mathrm{Sc} w_{23}\right] .
\end{aligned}
$$

The right-hand sides in the equations for $w_{3}$ and $p_{3}$ can be written in a similar form, with the coefficients of $\partial L_{1} / \partial T, L_{1}\left|L_{1}\right|^{2}$ and $L_{1}\left|L_{2}\right|^{2}$ given respectively by

$$
\begin{aligned}
& S_{w 0}=\frac{h(z)}{2 \mathrm{Sc}}, \\
& S_{w 3}=\frac{1}{2 \mathrm{Sc}}\left[3 h^{\prime} w_{21}+h w_{21}^{\prime}+2 q_{c}^{2} h Q_{1}\right], \\
& S_{w 4}=\frac{1}{4 \mathrm{Sc}}\left[5 h^{\prime} w_{23}+2 h w_{23}^{\prime}+3 q_{c}^{2} h Q_{3}\right], \\
& S_{p 0}=0, \\
& S_{p 3}=-\frac{1}{2 \mathrm{Sc}}\left[4 h^{\prime \prime} w_{21}+4 h^{\prime} w_{21}^{\prime}+h w_{21}^{\prime \prime}\right], \\
& S_{p 4}=-\frac{1}{4 \mathrm{Sc}}\left[6 h^{\prime \prime} w_{23}+7 h^{\prime} w_{23}^{\prime}+2 h w_{23}^{\prime \prime}\right] .
\end{aligned}
$$

Elimination of secular terms in the $O\left(\varepsilon^{3}\right)$ and $O\left(\varepsilon^{2}\right)$ problems via the solvability conditions (see Appendix) results in the following system of equations for the leading order amplitudes $L_{1}, L_{2}, L_{3}$

$$
\begin{aligned}
& C_{0} \frac{d L_{1}}{d T}=m C_{1} L_{1}+C_{2} L_{2}^{*} L_{3}^{*}+C_{3} L_{1}\left|L_{1}\right|^{2}+C_{4} L_{1}\left(\left|L_{2}\right|^{2}+\left|L_{3}\right|^{2}\right), \\
& C_{0} \frac{d L_{2}}{d T}=m C_{1} L_{2}+C_{2} L_{1}^{*} L_{3}^{*}+C_{3} L_{2}\left|L_{2}\right|^{2}+C_{4} L_{2}\left(\left|L_{1}\right|^{2}+\left|L_{3}\right|^{2}\right), \\
& C_{0} \frac{d L_{3}}{d T}=m C_{1} L_{3}+C_{2} L_{1}^{*} L_{2}^{*}+C_{3} L_{3}\left|L_{3}\right|^{2}+C_{4} L_{3}\left(\left|L_{1}\right|^{2}+\left|L_{2}\right|^{2}\right) .
\end{aligned}
$$

The coefficients $C_{j}$ for $j=0,2,3,4$ are given by

$$
C_{j}=-\frac{1}{2 q_{c}} \int_{-\infty}^{0} r_{j}(s) e^{q_{c} s} d s+M_{c} q_{c}^{2} \frac{2}{\zeta_{0} I_{\beta_{0}}^{\prime}\left(\zeta_{0}\right)} \int_{-\infty}^{0} I_{\beta_{0}}\left(\zeta_{0} e^{z / 2}\right)\left[\tilde{w}_{j}(z) A_{s}^{\prime}(z)+S_{A j}(z)\right] d z,
$$


with

$$
\tilde{w}_{j}(z)=\frac{1}{4 q_{c}^{3}} \int_{-\infty}^{0}\left(1+q_{c}|s-z|\right) r_{j}(s) e^{-q_{c}|s-z|} d s+\frac{e^{q_{c} z}}{4 q_{c}^{3}} \int_{-\infty}^{0}\left(-1+q_{c} s\right) r_{j}(s) e^{q_{c} s} d s,
$$

and

$$
r_{j}(z)=\frac{1}{\mathrm{Sc}}\left[S_{w j}^{\prime \prime}-q_{c}^{2} S_{w j}+S_{p j}^{\prime}\right]
$$

Finally,

$$
C_{1}=-q_{c}^{2} A_{10}(0)=\frac{q_{c}}{M_{c} \mathrm{Sc}} .
$$

In the case of small $\zeta_{0}$ these coefficients are particularly simple, and we restrict ourselves to this case. The coefficients $C_{j}, j=0,2,3,4$ are given in Appendix $\mathrm{C}$ as functions of $\mathrm{Sc}$ for several representative values of $\mu$. As seen from the appendix, for all parameter values that we considered $C_{0}>0$ and $C_{2}<0$. The latter inequality means that the hexagonal pattern corresponds to "down"-hexagons, i.e. such hexagonal convection cells in which the liquid is rising at the cell periphery and descending in the cell center. We note that in the case of usual Marangoni convection in a liquid layer heated from below, "down" hexagons appear for liquids with small Prandtl numbers $[7,8]$. In addition, $C_{1}>0$ as follows from the above formula. The behavior of the coefficients $C_{3}$ and $C_{4}$, which are mainly responsible for the appearance, stability and interaction of hexagons and stripes is more complex. We briefly list below well known general results concerning these patterns [25], and then relate these results to the problem at hand.

We consider steady states of the system (5.40), specifically, we are interested in the steady states that describe hexagons and stripes in the original system. Hexagonal patterns correspond to

$$
L_{1}=L_{2}=L_{3}=L_{h},
$$

where $L_{h}$ is a solution of the quadratic equation

$$
\left(C_{3}+2 C_{4}\right) L_{h}^{2}+C_{2} L_{h}+m C_{1}=0 .
$$

Stripes parallel to the $y$-axis correspond to

$$
L_{1}=L_{s}, \quad L_{2}=L_{3}=0, \quad L_{s}=\sqrt{-\frac{m C_{1}}{C_{3}}}
$$

We first discuss the stripes. The linear stability analysis of the system (5.40) in the case of stripes results in the following values for the growth rate $\sigma$ of perturbations:

$$
C_{0} \sigma_{1}=-2 m C_{1}, \quad \sigma_{2}=0, \quad C_{0} \sigma_{3,4}=C_{2} L_{s}-\left(C_{3}-C_{4}\right) L_{s}^{2}, \quad C_{0} \sigma_{5,6}=-C_{2} L_{s}-\left(C_{3}-C_{4}\right) L_{s}^{2} .
$$


Since the coefficients $C_{0}$ and $C_{1}$ are always positive, we conclude that supercritical stripes exist if $C_{3}<0$. They are stable if $C_{3}-C_{4}>0$ and the amplitude $L_{s}$ is sufficiently large, $L_{s}>\left|C_{2}\right| /\left(C_{3}-C_{4}\right)$.

The linear stability analysis of the system (5.40) in the case of hexagons results in the following values for the growth rate $\sigma$ of perturbations:

$$
\begin{aligned}
& C_{0} \sigma_{1}=C_{2} L_{h}+2\left(C_{3}+2 C_{4}\right) L_{h}^{2}, \quad C_{0} \sigma_{2,3}=2\left[-C_{2} L_{h}+\left(C_{3}-C_{4}\right) L_{h}^{2}\right], \\
& C_{0} \sigma_{4}=-3 C_{2} L_{h}, \quad \sigma_{5,6}=0 .
\end{aligned}
$$

Again, keeping in mind that $C_{0}>0, C_{1}>0$, we conclude that a necessary condition for stable hexagons to exist is $C_{3}+2 C_{4}<0$. This condition is not sufficient. If, in addition to this condition $C_{3}-C_{4}<0$, then the entire increasing branch of hexagons is stable. If the necessary condition is satisfied but $C_{3}-C_{4}>0$, hexagons can still be stable. Specifically, they are stable if $C_{3}+C_{4}<0$, but in this case only part of the increasing hexagon branch is stable. The hexagons in this case will coexist with stripes if $C_{3}<0$.

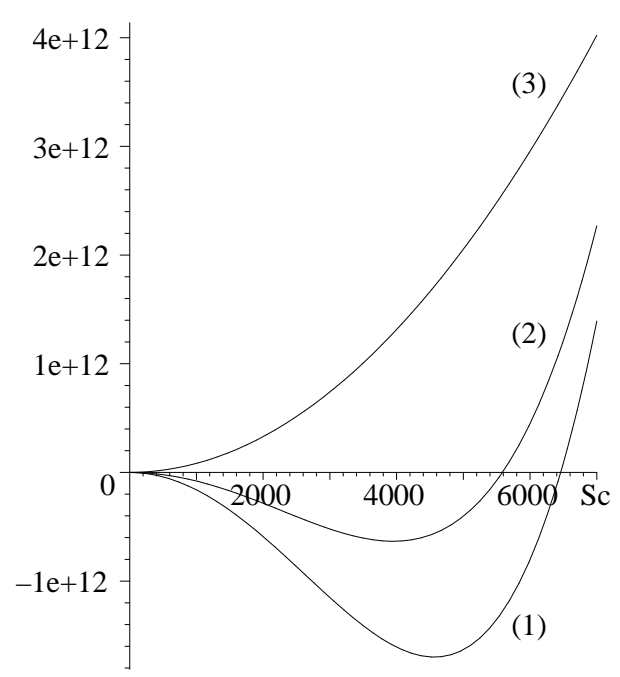

(a) $\mu=0.19$

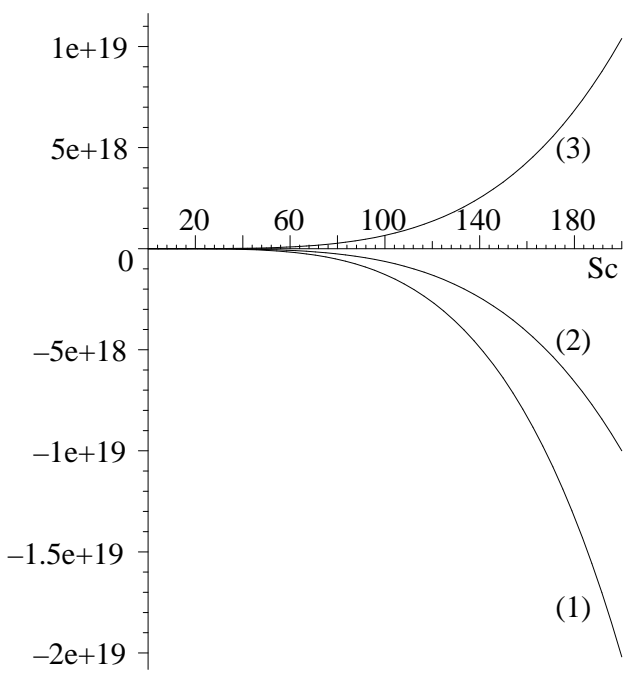

(b) $\mu=17.25$

Figure 5: Coefficients of the amplitude equations for two values of $\mu$. Here curves (1), (2) and (3) the graphs of $C_{3}+2 C_{4}, C_{3}+C_{4}$ and $C_{3}-C_{4}$, respectively (see text).

Applying these general results to the coefficients given in Figure 5 and Appendix $\mathrm{C}$ we can draw the following main conclusions.

For sufficiently small values of $\mu$ both stripes and hexagons can occur. The stripes are present and stable for an appreciable range of the Schmidt number, $0<\operatorname{Sc}<\operatorname{Sc}_{s}(\mu)$, where $\mathrm{Sc}_{s}(\mu)$ is an order one quantity. Hexagons exist for all $\mathrm{Sc}$ and thus there is a region of coexistence of hexagons and stripes. The hexagons are stable only if their amplitude $L_{h}$ is sufficiently small, i.e., when the Marangoni number is not too far from its critical value. 
For larger $\mu$ the stripes are still present. However, the ranges of Sc where the stripes exist and are stable become very small, and it is unlikely that the stripes can be observed in physical or numerical experiments. Hexagons exist and are stable for $\mathrm{Sc}>\operatorname{Sc}_{h}(\mu)$, where the critical number $\operatorname{Sc}_{h}(\mu)$ is typically quite small, $\operatorname{Sc}_{h}(\mu) \ll 1$, so that hexagons exist for all physically relevant values of Sc. The hexagons again are stable only if their amplitude $L_{h}$ is sufficiently small, i.e., when the Marangoni number is not too far from its critical value. The behavior of the system as the Marangoni number increases away from the critical value becomes strongly nonlinear and is not described by our weakly nonlinear analysis.

We can conclude that formation of hexagonal patterns with "down"-hexagons is the prevailing behavior of the system in the case of sufficiently small intensity of the light that triggers the photo-chemical reaction provided that the Marangoni number is sufficiently close to the instability threshold.

\section{Acknowledgment}

This work was supported by the DOE grant \#DE-FG02-03ER46069.

\section{References}

[1] A.A. Nepomnyashchy, M.G. Velarde, P. Colinet. Interfacial phenomena and convection. Chapman \& Hall/CRC, Boca Raton, FL, 2001.

[2] A. Nepomnyashchy, I. Simanovsky, J.C. Legros. Interfacial convection in multilayer systems. Springer, New York, NY, 2006.

[3] J.W. Scanlon, L.A. Segel. Finite amplitude cellular convection induced by surface tension. J. Fluid Mech., 30 (1967), 149-162.

[4] A. Cloot, G. Lebon. A nonlinear stability analysis of the Benard-Marangoni problem. J. Fluid Mech., 145 (1984), 447-469.

[5] J. Bragard, G. Lebon. Nonlinear Marangoni convection in a layer of finite depth. Europhys. Lett., 21 (1993), No. 8, 831-836.

[6] M. Bestehorn. Phase and amplitude instabilities for Benard-Marangoni convection in fluid layers with large aspect ratio. Phys. Rev. E, 48 (1993), No. 5, 3622-3634.

[7] A.A. Golovin, A.A. Nepomnyashchy, L.M. Pismen. Nonlinear evolution and secondary instabilities of Marangoni convection in a liquid-gas system with deformable interface. J. Fluid. Mech., 341 (1997), 317-341. 
[8] A.A. Golovin, A.A. Nepomnyashchy, L.M. Pismen. Pattern formation in long-scale Marangoni convection with deformable interface. Physica D, 81 (1995), No. 1-2, 117147.

[9] K. Eckert, M. Bestehorn, A. Thess. Square cells in surface-tension-driven Benard convection: experiment and theory. J. Fluid Mech., 356 (1998), 155-197.

[10] W.A. Tokaruk, T.C.A. Molteno, S.W. Morris. Benard-Marangoni convection in twolayered liquids. Phys. Rev. Lett., 84 (2000), No. 16, 3590-3593.

[11] J. Lucassen. Longitudinal capillary waves. 1. Theory. Trans. Farad. Soc., 64 (1968), No. 548P, 2221-2229.

[12] R.D. Benguria, M.C. Depassier. On the linear-stability theory of Benard-Marangoni convection. Phys. Fluids A, 1 (1989), No. 7, 1123-1127.

[13] A.A. Nepomnyashchy, M.G. Velarde. A 3-dimensional description of solitary waves and their interaction in Marangoni-Benard layers. Phys. Fluids, 6 (1994), No. 1, 187-198.

[14] M.G. Velarde, A.A. Nepomnyashchy, M. Hennenberg. Onset of oscillatory interfacial instability and wave motions in Benard layers. Adv. Appl. Mech., 37 (2001), 167-238.

[15] M.G. Velarde. Benard layers, overstability, and waves. Springer Tracts in Modern Physics, 207 (2006), 129-145.

[16] W.B. Zimmerman, J.M. Rees, B.N. Hewakandamby. Numerical analysis of solutocapillary Marangoni-induced interfacial waves. Adv. Colloid Interface Sci., 134-135 (2007), 346-359.

[17] B.A. Bezuglyi, V.S. Maiorov. Role of the surface forces during the image-formation on thin liquid-films. Zh. Nauch. Priklad. Photogr., 26 (1981), No. 6, 422-429.

[18] B.A. Bezuglyi, D.P. Krindach, V.S. Maiorov. Recovery of the representation in liquidfilms with utilizations of occurring of thermocapillary convections. Zh. Tekhn. Fiz., 52 (1982), No. 12, 2416-2418.

[19] B.A. Bezuglyi. Intensification of photoinduced capillary convection in evaporating fluid layer. Pisma Zh. Tekhn. Fiz., 17 (1991), No. 21, 49-53.

[20] D. Semwogerere, M.F. Schatz. Evolution of hexagonal patterns from controlled initial conditions in a Benard-Marangoni convection experiment. Phys. Rev. Lett., 88 (2002), No. 5, art. 054501.

[21] N. Garnier, R.O. Grigoriev, M.F. Schatz. Optical manipulation of microscale fluid flow. Phys. Rev. Lett., 91 (2003), No. 5, art. 054501. 
[22] E. Kumacheva, L. Li, M.A. Winnik, D.M. Shinozaki, P.C. Cheng. Direct imaging of surface and bulk structures in solvent cast polymer blend films. Langmuir, 13 (1997), No. 9, 2483-2489.

[23] Y. Inaki, Y. Wang, T. Saito, K. Takemoto. Reversible photochemical reactions of thymines having long alkyl chain. J. Photopolymer Sci. Technol., 5 (1992), No. 3, 567568 .

[24] Chemical Reviews, 2000, Vol. 100, issue 5: Photochromism: Memories and Switches Thematic Issue.

[25] D. Walgraef. Spatio-temporal pattern formation. Springer, New York, NY, 1996.

\section{Appendix A. Solution of the $O\left(\varepsilon^{2}\right)$ problem}

The $z$-dependent coefficients of $E_{1}, E_{2}$ and $E_{3}$ in the solution $p_{2}$ of the $O\left(\varepsilon^{2}\right)$ problem are given by

$$
\begin{aligned}
& p_{21}=\frac{1}{16 q_{c}^{2} \mathrm{Sc}^{2}}\left(b_{1}-2 q_{c} z\right) e^{2 q_{c} z}, \\
& p_{22}=-\frac{z^{2}}{4 \mathrm{Sc}^{2}} e^{2 q_{c} z}, \\
& p_{23}=-\frac{1}{8 q_{c}^{2} \mathrm{Sc}^{2}}\left(27-6 q_{c} z+q_{c}^{2} z^{2}\right) e^{2 q_{c} z}+\frac{1}{q_{c}^{2} \mathrm{Sc}^{2}} b_{3} e^{q_{c} z \sqrt{3}} .
\end{aligned}
$$

The $z$-dependent coefficients of $E_{1}, E_{2}$ and $E_{3}$ in the solution $w_{2}$ of the $O\left(\varepsilon^{2}\right)$ problem are given by

$$
\begin{aligned}
& w_{21}=-\frac{1}{64 q_{c}^{3} \mathrm{Sc}^{3}}\left[q_{c} z\left(1-2 b_{1}\right)+2 q_{c}^{2} z^{2}\right] e^{2 q_{c} z}, \\
& w_{22}=0, \\
& w_{23}=\frac{1}{2 q_{c}^{3} \mathrm{Sc}^{3}}\left[\left(-24+3 q_{c} z\right) e^{2 q_{c} z}+\left(24+b_{3} q_{c} z\right) e^{q_{c} z \sqrt{3}}\right] .
\end{aligned}
$$

Next, the coefficients in $A_{2}$ are determined by the equations

$$
\begin{aligned}
& A_{21}^{0 \prime}-\left(4 q_{c}^{2}+\hat{k}_{1} e^{z}+\hat{k}_{2}\right) A_{21}^{0}=F_{21}^{0}, \quad A_{21}^{0}{ }^{\prime}(0)=0, \quad A_{21}^{0}(-\infty)=0, \\
& A_{21}^{1}{ }^{\prime \prime}-\left(4 q_{c}^{2}+\hat{k}_{1} e^{z}+\hat{k}_{2}\right) A_{21}^{1}=F_{21}^{1}, \quad A_{21}^{1}{ }^{\prime}(0)=0, \quad A_{21}^{1}(-\infty)=0, \\
& A_{22}^{0 \prime}-\left(\hat{k}_{1} e^{z}+\hat{k}_{2}\right) A_{22}^{0}=F_{22}^{0}, \quad A_{22}^{0}{ }^{\prime}(0)=0, \quad A_{22}^{0}(-\infty)=0, \quad A_{22}^{1}(z) \equiv 0, \\
& A_{23}^{0 \prime \prime}-\left(3 q_{c}^{2}+\hat{k}_{1} e^{z}+\hat{k}_{2}\right) A_{23}^{0}=F_{23}^{0}, \quad A_{23}^{0}{ }^{\prime}(0)=0, \quad A_{23}^{0}(-\infty)=0,
\end{aligned}
$$




$$
A_{23}^{1 \prime \prime}-\left(3 q_{c}^{2}+\hat{k}_{1} e^{z}+\hat{k}_{2}\right) A_{23}^{1}=F_{23}^{1}, \quad A_{23}^{1}{ }^{\prime}(0)=0, \quad A_{23}^{1}(-\infty)=0,
$$

where

$$
A_{2 j}=A_{2 j}^{0}+b_{j} A_{2 j}^{1}, \quad j=1,2,3
$$

and

$$
\begin{aligned}
& F_{21}^{0}=-\frac{q_{c} z+2 q_{c}^{2} z^{2}}{64 q_{c}^{3} \mathrm{Sc}^{3}} A_{s}^{\prime}(z) e^{2 q_{c} z}-\frac{1}{2 \mathrm{Sc}} h^{\prime}(z) A_{10}(z)+\frac{1}{2 \mathrm{Sc}} h(z) A_{10}^{\prime}(z), \\
& F_{21}^{1}=\frac{1}{32 q_{c}^{3} \mathrm{Sc}^{3}} A_{s}^{\prime}(z) q_{c} z e^{2 q_{c} z}, \\
& F_{22}^{0}=\frac{1}{2 \mathrm{Sc}} h^{\prime}(z) A_{10}(z)+\frac{1}{2 \mathrm{Sc}} h(z) A_{10}^{\prime}(z), \quad F_{22}^{1} \equiv 0, \\
& F_{23}^{0}=\frac{1}{2 q_{c}^{3} \mathrm{Sc}^{3}}\left[\left(-24+3 q_{c} z\right) e^{2 q_{c} z}+24 e^{q_{c} z \sqrt{3}}\right] A_{s}^{\prime}(z)-\frac{1}{2 \mathrm{Sc}} h^{\prime}(z) A_{10}(z)+\frac{1}{\mathrm{Sc}} h(z) A_{10}^{\prime}(z), \\
& F_{23}^{1}=\frac{1}{2 q_{c}^{3} \mathrm{Sc}^{3}} A_{s}^{\prime}(z) q_{c} z e^{q_{c} z \sqrt{3}} .
\end{aligned}
$$

Making the same change of variables $A_{2 j}^{0,1}(z)=a_{2 j}^{0,1}(\zeta), j=1,2,3$ as before in each of the equations, we obtain the solutions in terms of Bessel functions as

$a_{2 j}^{0,1}(\zeta)=C_{2 j}^{0,1} I_{\beta_{2 j}}\left(\zeta_{0} \zeta\right)-I_{\beta_{2 j}}\left(\zeta_{0} \zeta\right) \int_{\zeta}^{1} \frac{1}{s} K_{\beta_{2 j}}\left(\zeta_{0} s\right) f_{2 j}^{0,1}(s) d s-K_{\beta_{2 j}}\left(\zeta_{0} \zeta\right) \int_{0}^{\zeta} \frac{1}{s} I_{\beta_{2 j}}\left(\zeta_{0} s\right) f_{2 j}^{0,1}(s) d s$.

Here

$$
f_{2 j}^{0,1}(\zeta)=4 F_{2 j}^{0,1}(z), \quad j=1,2,3,
$$

$C_{2 j}^{0,1}$ are constants given as

$$
C_{2 j}^{0,1}=\frac{K_{\beta_{2 j}}^{\prime}\left(\zeta_{0}\right)}{I_{\beta_{2 j}}^{\prime}\left(\zeta_{0}\right)} \int_{0}^{1} \frac{1}{s} I_{\beta_{2 j}}\left(\zeta_{0} s\right) f_{2 j}^{0,1}(s) d s
$$

and

$$
\beta_{21}^{2}=16 q_{c}^{2}+4 \hat{k}_{2}, \quad \beta_{22}^{2}=4 \hat{k}_{2}, \quad \beta_{23}^{2}=12 q_{c}^{2}+4 \hat{k}_{2} .
$$

Now we determine the constants $b_{1}$ and $b_{2}$ using the boundary condition

$$
z=0: \quad \frac{\partial^{2} w_{2}}{\partial z^{2}}=M_{c} \nabla_{x y}^{2} A_{2}
$$


to find

$$
b_{1}=\frac{1-32 q_{c}^{3} \mathrm{Sc}^{3} M_{c} a_{21}^{0}(1)}{1+2 q_{c} \mathrm{Sc} M_{c} a_{21}^{1}(1)}, \quad b_{3}=\frac{2-q_{c}^{3} \mathrm{Sc}^{3} M_{c} a_{23}^{0}(1)}{\sqrt{3} / 3+q_{c} \mathrm{Sc} M_{c} a_{23}^{1}(1)} .
$$

Here $a_{2 j}^{0,1}(1)$ are calculated from (5.41) as

$$
a_{2 j}^{0,1}(1)=-\frac{1}{\zeta_{0} I_{\beta_{2 j}}^{\prime}\left(\zeta_{0}\right)} \int_{0}^{1} \frac{1}{s} I_{\beta_{2 j}}\left(\zeta_{0} s\right) f_{2 j}^{0,1}(s) d s .
$$

To complete the solution of the $O\left(\varepsilon^{2}\right)$ problem we present the expressions for $u_{2}$ and $v_{2}$. They are given by

$$
u_{2}=\frac{\partial Q}{\partial x}, \quad v_{2}=\frac{\partial Q}{\partial y}
$$

where $Q$ is the solution of the problem

$$
\begin{aligned}
& \nabla^{2} Q=\frac{p_{2}}{\mathrm{Sc}}+\frac{e^{2 q_{c} z}}{8 q_{c}^{2} \mathrm{Sc}^{3}}\left[-E_{1}+\left(1+4 q_{c} z+2 q_{c}^{2} z^{2}\right) E_{2}+\left(-1+2 q_{c} z+q_{c}^{2} z^{2}\right) E_{3}\right] \\
& z=0: \quad \frac{\partial Q}{\partial z}=-M_{c} A_{2}, \quad z=-\infty: \quad Q=0 .
\end{aligned}
$$

It has the form

$$
Q=Q_{1} E_{1}+Q_{2} E_{2}+Q_{3} E_{3}
$$

with

$$
\begin{aligned}
& Q_{1}=\frac{1}{256 q_{c}^{4} \mathrm{Sc}^{3}}\left[-2\left(3 q_{c} z+2 q_{c}^{2} z^{2}\right)+4 q_{c} z b_{1}+d_{1}\right] e^{2 q_{c} z}, \\
& Q_{2}=\frac{1}{32 q_{c}^{4} \mathrm{Sc}^{3}}\left(-3+4 q_{c} z\right) e^{2 q_{c} z}, \\
& Q_{3}=\frac{1}{6 q_{c}^{4} \mathrm{Sc}^{3}}\left\{3\left(-15+2 q_{c} z\right) e^{2 q_{c} z}+b_{3} q_{c} z \sqrt{3} e^{q_{c} z \sqrt{3}}+d_{3} e^{q_{c} z \sqrt{3}}\right\}, \\
& d_{1}=3-128 q_{c}^{3} \mathrm{Sc}^{3} M_{c} a_{21}^{0}(1)-2\left[1+4 q_{c} \mathrm{Sc}_{c} a_{21}^{1}(1)\right] b_{1}, \\
& d_{3}=2 \sqrt{3}\left[14-q_{c}^{3} \mathrm{Sc}^{3} M_{c} a_{23}^{0}(1)\right]-b_{3}\left[1+2 \sqrt{3} q_{c} \mathrm{Sc}_{c} a_{23}^{1}(1)\right] .
\end{aligned}
$$




\section{Appendix B. Solvability conditions}

Consider the system of equations

$$
\begin{aligned}
& \nabla^{2} A-\left(\hat{k}_{1} e^{z}+\hat{k}_{2}\right) A-w \frac{d A_{s}}{d z}=R_{A}, \\
& \operatorname{Sc} \nabla^{2} w-\frac{\partial p}{\partial z}=R_{w}, \\
& \nabla^{2} p=R_{p} .
\end{aligned}
$$

with the boundary conditions

$$
\begin{aligned}
& z=0: \frac{\partial A}{\partial z}=0, \quad w=0, \quad \frac{\partial^{2} w}{\partial z^{2}}=M_{c} \nabla_{x y}^{2} A+R_{b}, \\
& z=-\infty: \quad A=0, \quad w=0 .
\end{aligned}
$$

In order to derive solvability conditions for the system of equations, we proceed as follows. Instead of deriving the adjoint problem and orthogonality conditions, we assume that the right hand sides of the equations contain secular terms, specifically, that they have the form

$$
R_{A}=r_{A}(z) e_{j}, \quad R_{w}=r_{w}(z) e_{j}, \quad R_{p}=r_{p}(z) e_{j}, \quad R_{b}=r_{b} e_{j}
$$

where $j$ is 1,2 or 3 , and attempt to solve the system, which results in solvability conditions. We seek solution in the form

$$
A=\tilde{A}(z) e_{j}, \quad w=\tilde{w}(z) e_{j}, \quad p=\tilde{p}(z) e_{j},
$$

to obtain

$$
\begin{aligned}
& \tilde{A}^{\prime \prime}-\left(q_{c}^{2}+\hat{k}_{1} e^{z}+\hat{k}_{2}\right) \tilde{A}-\tilde{w} A_{s}^{\prime}=r_{A}, \\
& \operatorname{Sc}\left(\tilde{w}^{\prime \prime}-q_{c}^{2} \tilde{w}\right)-\tilde{p}^{\prime}=r_{w}, \\
& \tilde{p}^{\prime \prime}-q_{c}^{2} \tilde{p}=r_{p}, \\
& z=0: \quad \tilde{A}^{\prime}=0, \quad \tilde{w}=0, \quad \tilde{w}^{\prime \prime}=-M_{c} q_{c}^{2} \tilde{A}+r_{b}, \\
& z=-\infty: \quad \tilde{A}=0, \quad \tilde{w}=0 .
\end{aligned}
$$

We can eliminate $\tilde{p}(z)$ from the last two equations in (5.44) thus reducing them to a single fourth order equation for $\tilde{w}$

$$
\tilde{w}^{(\mathrm{IV})}-2 q_{c}^{2} \tilde{w}^{\prime \prime}+q_{c}^{4} \tilde{w}=r(z),
$$

where

$$
r(z)=\frac{1}{\mathrm{Sc}}\left[r_{w}^{\prime \prime}-q_{c}^{2} r_{w}+r_{p}^{\prime}\right] .
$$


The solution of this equation that satisfies the boundary conditions

$$
\tilde{w}(0)=\tilde{w}(-\infty)=0,
$$

is

$$
\tilde{w}(z)=\frac{1}{4 q_{c}^{3}} \int_{-\infty}^{0}\left(1+q_{c}|s-z|\right) r(s) e^{-q_{c}|s-z|} d s+\frac{e^{q_{c} z}}{4 q_{c}^{3}} \int_{-\infty}^{0}\left(-1+q_{c} s\right) r(s) e^{q_{c} s} d s .
$$

We remark that the solution is not unique - the function $c z \exp \left(q_{c} z\right)$ where $c$ is an arbitrary constant can be added to the solution. However, this function disappears from the solvability condition so that we can disregard this part of the solution, i.e. take $c=0$. The quantity $\tilde{w}^{\prime \prime}(0)$ that is needed in the sequel, can be computed from (5.46) as

$$
\tilde{w}^{\prime \prime}(0)=\frac{1}{2 q_{c}} \int_{-\infty}^{0} r(s) e^{q_{c} s} d s
$$

Now we turn to the equation (5.44a) for $\tilde{A}$. The equation can be solved by reducing it to a Bessel equation, an approach used several times in this paper. We do not present the details of this calculation (cf. Section 4) but rather give the final answer, which is the value of the solution at $z=0$ :

$$
\tilde{A}(0)=-\frac{2}{\zeta_{0} I_{\beta_{0}}^{\prime}\left(\zeta_{0}\right)} \int_{-\infty}^{0} I_{\beta_{0}}\left(\zeta_{0} e^{z / 2}\right)\left[\tilde{w}(z) A_{s}^{\prime}(z)+r_{A}(z)\right] d z
$$

Substituting (5.47), (5.48) into the last condition in (5.45a) gives the solvability condition

$$
\frac{1}{2 q_{c}} \int_{-\infty}^{0} r(s) e^{q_{c} s} d s=M_{c} q_{c}^{2} \frac{2}{\zeta_{0} I_{\beta_{0}}^{\prime}\left(\zeta_{0}\right)} \int_{-\infty}^{0} I_{\beta_{0}}\left(\zeta_{0} e^{z / 2}\right)\left[\tilde{w}(z) A_{s}^{\prime}(z)+r_{A}(z)\right] d z+r_{b}
$$

\section{Appendix C. Coefficients of the amplitude equations}

$$
\begin{aligned}
& \mu=17.25: \\
& \mathrm{Sc}^{2} C_{0}=0.263+0.053 \mathrm{Sc}, \quad \mathrm{Sc}^{3} C_{2}=-0.043-0.0205 \mathrm{Sc}, \\
& \mathrm{Sc}^{5} C_{3}=0.0006-57 \mathrm{Sc}-186 \mathrm{Sc}^{2}+1.25 \cdot 10^{8} \mathrm{Sc}^{4} \\
& \mathrm{Sc}^{5} C_{4}=10.45-1.45 \cdot 10^{10} \mathrm{Sc}^{3}+5.43 \mathrm{Sc}-0.059 \mathrm{Sc}^{2}-6.30 \cdot 10^{9} \mathrm{Sc}^{4} \\
& \mu=13.15: \\
& \mathrm{Sc}^{2} C_{0}=0.34+0.07 \mathrm{Sc}, \quad \mathrm{Sc}^{3} C_{2}=-0.056-0.026 \mathrm{Sc}
\end{aligned}
$$




$$
\begin{aligned}
& \mathrm{Sc}^{5} C_{3}=0.0011-12.51 \mathrm{Sc}-47.86 \mathrm{Sc}^{2}+1.2 \cdot 10^{7} \mathrm{Sc}^{4}, \\
& \mathrm{Sc}^{5} C_{4}=24.79-1.39 \cdot 10^{9} \mathrm{Sc}^{3}+13.58 \mathrm{Sc}-10.81 \mathrm{Sc}^{2}-6 \cdot 10^{8} \mathrm{Sc}^{4} \text {; } \\
& \mu=10.56 \text { : } \\
& \mathrm{Sc}^{2} C_{0}=0.42+0.088 \mathrm{Sc}, \quad \mathrm{Sc}^{3} C_{2}=-0.069-0.032 \mathrm{Sc}, \\
& \mathrm{Sc}^{5} C_{3}=0.002-3.83 \mathrm{Sc}+1687 \mathrm{Sc}^{2}+1.88 \cdot 10^{6} \mathrm{Sc}^{4} \text {, } \\
& \mathrm{Sc}^{5} C_{4}=51.56-2.1 \cdot 10^{8} \mathrm{Sc}^{3}+31 \mathrm{Sc}+1700 \mathrm{Sc}^{2}-9.1 \cdot 10^{7} \mathrm{Sc}^{4} \text {; } \\
& \mu=8.76 \text { : } \\
& \mathrm{Sc}^{2} C_{0}=0.497+0.105 \mathrm{Sc}, \quad \mathrm{Sc}^{3} C_{2}=-0.082-0.038 \mathrm{Sc}, \\
& \mathrm{Sc}^{5} C_{3}=-1.45 \mathrm{Sc}+0.0032+12.7 \mathrm{Sc}^{2}+3.9 \cdot 10^{5} \mathrm{Sc}^{4} \text {, } \\
& \mathrm{Sc}^{5} C_{4}=97.6-4.3 \cdot 10^{7} \mathrm{Sc}^{3}+73.4 \mathrm{Sc}+27.7 \mathrm{Sc}^{2}-1.9 \cdot 10^{7} \mathrm{Sc}^{4} \text {; } \\
& \mu=7.45 \text { : } \\
& \mathrm{Sc}^{2} C_{0}=0.58+0.124 \mathrm{Sc}, \quad \mathrm{Sc}^{3} C_{2}=-0.0953-0.044 \mathrm{Sc}, \\
& \mathrm{Sc}^{5} C_{3}=-0.78 \mathrm{Sc}+0.0051+1.01 \cdot 10^{5} \mathrm{Sc}^{4}+4.50 \mathrm{Sc}^{2}, \\
& \mathrm{Sc}^{5} C_{4}=166-1.09 \cdot 10^{7} \mathrm{Sc}^{3}+215 \mathrm{Sc}-4.7 \cdot 10^{6} \mathrm{Sc}^{4}+66.8 \mathrm{Sc}^{2} \text {; } \\
& \mu=5.66 \text { : } \\
& \mathrm{Sc}^{2} C_{0}=0.74+0.16 \mathrm{Sc}, \quad \mathrm{Sc}^{3} C_{2}=-0.12-0.055 \mathrm{Sc}, \\
& \mathrm{Sc}^{5} C_{3}=0.014-2.35 \mathrm{Sc}+2.44 \mathrm{Sc}^{2}+1.05 \cdot 10^{4} \mathrm{Sc}^{4} \text {, } \\
& \mathrm{Sc}^{5} C_{4}=-3379-1.1 \cdot 10^{6} \mathrm{Sc}^{3}+2.7 \cdot 10^{4} \mathrm{Sc}+1.3 \cdot 10^{4} \mathrm{Sc}^{2}-4.7 \cdot 10^{5} \mathrm{Sc}^{4} \text {; } \\
& \mu=2.59 \text { : } \\
& \mathrm{Sc}^{2} C_{0}=1.47+0.35 \mathrm{Sc}, \quad \mathrm{Sc}^{3} C_{2}=-0.25-0.10 \mathrm{Sc}, \\
& \mathrm{Sc}^{5} C_{3}=0.053-15.07 \mathrm{Sc}+0.67 \mathrm{Sc}^{2}+23 \mathrm{Sc}^{4} \text {, } \\
& \mathrm{Sc}^{5} C_{4}=12468-2077 \mathrm{Sc}^{3}+5115 \mathrm{Sc}-154 \mathrm{Sc}^{2}-890 \mathrm{Sc}^{4}
\end{aligned}
$$


$\mu=1.45$ :

$\mathrm{Sc}^{2} C_{0}=2.39+0.613 \mathrm{Sc}, \quad \mathrm{Sc}^{3} C_{2}=-.41-.155 \mathrm{Sc} ;$

$\mathrm{Sc}^{5} C_{3}=0.25-18.95 \mathrm{Sc}+8.98 \mathrm{Sc}^{2}+0.46 \mathrm{Sc}^{4}$,

$\mathrm{Sc}^{5} C_{4}=-31195-16178 \mathrm{Sc}-25.6 \mathrm{Sc}^{3}-1096 \mathrm{Sc}^{2}-10.7 \mathrm{Sc}^{4}$,

$\mu=0.86$ :

$\mathrm{Sc}^{2} C_{0}=3.74+1.02 \mathrm{Sc}, \quad \mathrm{Sc}^{3} C_{2}=-0.65-0.23 \mathrm{Sc}$,

$\mathrm{Sc}^{5} C_{3}=-46.6 \mathrm{Sc}+0.96+15.4 \mathrm{Sc}^{2}+0.046 \mathrm{Sc}^{4}$,

$\mathrm{Sc}^{5} C_{4}=-45494-0.54 \mathrm{Sc}^{3}-24623 \mathrm{Sc}-2089 \mathrm{Sc}^{2}-0.19 \mathrm{Sc}^{4}$;

$\mu=0.48$ :

$\mathrm{Sc}^{2} C_{0}=6.19+1.8 \mathrm{Sc}, \quad \mathrm{Sc}^{3} C_{2}=-1.09-0.35 \mathrm{Sc}$,

$\mathrm{Sc}^{5} C_{3}=-172 \mathrm{Sc}+4.49+47.5 \mathrm{Sc}^{2}+0.0099 \mathrm{Sc}^{4}$,

$\mathrm{Sc}^{5} C_{4}=-139054-0.0089 \mathrm{Sc}^{3}-76636 \mathrm{Sc}-7155 \mathrm{Sc}^{2}+0.0061 \mathrm{Sc}^{4}$;

$\mu=0.19$ :

$\mathrm{Sc}^{2} C_{0}=14.5+4.47 \mathrm{Sc}, \quad \mathrm{Sc}^{3} C_{2}=-2.6-0.78 \mathrm{Sc}$,

$\mathrm{Sc}^{5} C_{3}=58.97-2012.7 \mathrm{Sc}+0.0013 \mathrm{Sc}^{4}+490.2 \mathrm{Sc}^{2}$,

$\mathrm{Sc}^{5} C_{4}=-146558-0.00001 \mathrm{Sc}^{3}-816695 \mathrm{Sc}+0.0013 \mathrm{Sc}^{4}-81469 \mathrm{Sc}^{2}$. 